\title{
Trend Analysis of Hospital Resource Utilization for Prolonged Mechanical Ventilation Patients in Taiwan: A Population-Based Study
}

\author{
Tien-Chiung Hung MSc, Yung-Fa Lai MD, Ching-Wan Tseng MSc, \\ Yong-Han Hong PhD, and Hon-Yi Shi MPH DrPH
}

\begin{abstract}
BACKGROUND: The aging Taiwan population is expected to require vast medical resources, including prolonged mechanical ventilation (PMV). This study determined the trends in hospital resource utilization and associated factors in PMV patients in Taiwan. METHODS: All patients who had received mechanical ventilation for $>21$ days (International Classification of Diseases, 9th Revision, Clinical Modification codes 518.81-518.89) during 2004-2007 were recruited to the study. Administrative claims data obtained from the Bureau of National Health Insurance of Taiwan were analyzed. RESULTS: The study analyzed 65,181 patients who had received PMV during 20042007. The number of PMVs per 100,000 persons was 94.30 in 2004, and it gradually decreased to 89.38 in 2007 , which was a change rate of $\mathbf{- 5 . 2 2 \%}$. During the study period, stay significantly decreased, from 35.12 days to 31.61 days, whereas hospital treatment costs significantly increased, from $\$ 7,933.17$ to $\$ 8,257.52(P<.001)$. Considerably decreased stay and increased hospital treatment costs were significantly associated with age, number of comorbidities, hospital level, hospital volume, and patient referral source $(P<.001)$. CONCLUSIONS: These population-based data demonstrated a decrease in the prevalence of PMV, especially for older patients, and that stay decreased; however, hospital treatment costs increased. Moreover, healthcare providers and patients should recognize that attributes of both the patient and the hospital may affect hospital resource utilization. Additionally, these analytical results should be applicable to similar populations in other countries. Key words: prolonged mechanical ventilation; trends; predictors; hospital resource utilization. [Respir Care 2013;58(4):669-675. () 2013 Daedalus Enterprises]
\end{abstract}

\section{Introduction}

Patients admitted to ICUs often require mechanical ventilation. ${ }^{1,2}$ Many critically ill patients have persistent respiratory failure that requires prolonged mechanical ventilation (PMV). PMV is commonly defined as more than 21 days of mechanical ventilation lasting at least 6 hours per day, although mechanical ventilation for a shorter du-

Mr Hung is affiliated with the Department of Respiratory Therapy, E-Da Hospital; Dr Lai is affiliated with the Division of Pulmonary Medicine, Department of Internal Medicine, E-Da Hospital and with the Department of Occupational Therapy, I-Shou University; Mr Tseng is affiliated with the Department of Respiratory Therapy, Kaohsiung Chang Gung Memorial Hospital; Dr Hong is affiliated with the Department of Medical Nutrition, I-Shou University; and Dr Shi is affiliated with the Graduate Institute of Healthcare Administration, Kaohsiung Medical University, Kaohsiung City, Taiwan, Republic of China.

The authors have disclosed no conflicts of interest. ration has also been mentioned in the literature. ${ }^{1-3}$ The Centers for Medicare and Medicaid Services in the United States define PMV as at least 21 continuous days of mechanical ventilation for at least 6 hours per day. ${ }^{3}$ Indeed, most ventilated patients transferred to long-term acute care hospitals have received mechanical ventilation for at least 21 days. $^{3}$ Of all patients who receive mechanical ventilation, an estimated 5-13\% required PMV. ${ }^{3,4}$ Although PMV patients consume substantial medical resources, their outcomes tend to be poor, especially in the rapidly growing elderly population. ${ }^{2-5}$

\footnotetext{
Correspondence: Hon-Yi Shi MPH DrPH, Graduate Institute of Healthcare Administration, Kaohsiung Medical University, 100 Shih-Chun First Road, Kaohsiung City, Taiwan, Republic of China. E-mail: hshi@kmu.edu.tw.
}

DOI: $10.4187 /$ respcare.01519 
Historically, patients requiring PMV tend to be treated in ICUs, and associated discharge rates are low. However in the 1990s, changes in the medical care reimbursement regulations of the Taiwan healthcare system created incentives to transfer patients requiring PMV from ICUs of acute care hospitals to long-term assisted care facilities..$^{2-6}$ The policy for PMV patients implemented by the Taiwan Bureau of National Health Insurance (BNHI) in 2000 required the transfer of such patients to a respiratory care center after an ICU stay of 21 days, and to a respiratory care ward after a respiratory care center stay of 42 days. $^{7}$

Although many studies have evaluated PMV outcomes, few longitudinal studies have exceeded 2 years, ${ }^{1-5}$ and most published data are limited to United States or European populations. ${ }^{1-6}$ Therefore, the findings of previous studies of PMV outcomes may not be generalizable to patient populations in Asian countries. Further, no studies have longitudinally evaluated temporal trends in hospital resource utilization by PMV patients, and none have systematically evaluated associations with hospital resource utilization in this patient group. ${ }^{8}$

Clinical decision making and policy making for PMV is challenging and requires efficient planning and effective medical care. Thus, the aim of this population-based study was to analyze trends in hospital resource utilization and associated factors in a population of patients who had received PMV.

\section{Methods}

\section{Study Design and Data Sources}

This study analyzed administrative claims data obtained from the BNHI. Because the BNHI is the sole payer in Taiwan, the data set included information for all patients discharged from all hospitals in Taiwan during the study period, and was assumedly the most comprehensive and reliable data source for the study. This study analyzed data for all patients who had received mechanical ventilation or PMV (International Classification of Diseases, 9th Revision, Clinical Modification [ICD-9-CM] disposition codes 518.81-518.89 and ICD-9-CM 96.72, respectively). After excluding data for patients younger than 17 years $(351$ subjects), the final data set included data for 65,181 PMV patients treated from 2004 to 2007 . The study protocol was approved by the institutional review board of E-Da Hospital.

\section{Patients and Variables}

The following attributes of this Taiwan population of PMV patients were analyzed: age, sex, comorbidity, hospital level, hospital volume, and patient referral source. Age categories were $<65$ years, 65-74 years, and

\section{QUICK LOOK}

\section{Current knowledge}

Prolonged mechanical ventilation (PMV) is defined as $>21$ days of ventilatory support. The number of patients receiving PMV in North America has been rapidly increasing. The rate of PMV in other countries is not well described.

\section{What this paper contributes to our knowledge}

In Taiwan, from 2004 through 2007, the requirement for PMV fell by 5\%, hospital costs increased, and hospital stay decreased.

$\geq 75$ years. Comorbidities were identified from ICD-9-CM codes for primary and secondary diagnoses, excluding cancer related codes. These ICD-9-CM codes were used to calculate the Deyo-Charlson comorbidity index. ${ }^{9-12}$ Patient referral source was categorized by hospital department as general medicine, general surgery, cardiology, pulmonary, neurology/neurosurgery, and other. Hospital level was accredited by the Taiwan Joint Commission on Hospital Accreditation as medical center ( $>500$ beds), regional hospital ( $>300$ beds), or district hospital ( $>100$ beds). The "selective referral hypothesis" postulates that the severity of PMV at admission may differ between high-volume and low-volume hospitals. Because official performance information to help PMV patients select healthcare providers is not available, patients choose hospitals with better reputations or more successful surgeries after consulting with their relatives and friends. Hospital volume was categorized as low, medium, and high if the number of patients treated at the hospital in the previous year was $\leq 25 \%$, $26-74 \%$, and $\geq 75 \%$, respectively, of the total patients treated at the hospital that year. Hospital resource utilization was measured by stay and hospital treatment cost.

\section{Statistical Analyses}

Continuous variables were tested for statistical significance by one-way analysis of variance, and categorical variables were tested by chi-square analysis. Analysis of trends in the prevalence of PMV patients was assessed by Cochrane-Armitage trend test. Hospital treatment costs were analyzed by collecting data for the following medical costs, which are required data in standard administrative claims for reimbursement from the Taiwan BNHI: operating room, radiology, physical therapy, hospital room, anesthetist, pharmacy, laboratory, special materials, surgeon, and others. Multiple regression models used to predict stay and hospital treatment costs included both patient attributes and hospital attributes. 
Trend Analysis of Hospital Resource Utilization for Prolonged Mechanical Ventilation

Table 1. Prevalence of Prolonged Mechanical Ventilation, 2004 Through 2007

\begin{tabular}{ccccc}
\hline \hline Year & $\begin{array}{c}\text { Total } \\
\text { Population }\end{array}$ & $\begin{array}{c}\text { PMV } \\
\text { Patients }\end{array}$ & $\begin{array}{c}\text { Prevalence } \\
(1 / 100,000 \text { persons })\end{array}$ & $\begin{array}{c}\text { Change Rate } \\
(\%)^{*}\end{array}$ \\
\hline 2004 & $17,281,468$ & 16,297 & 94.30 & Reference \\
2005 & $17,559,586$ & 16,278 & 92.70 & -1.70 \\
2006 & $17,823,777$ & 16,310 & 91.51 & -2.97 \\
2007 & $18,232,185$ & 16,296 & 89.38 & -5.22
\end{tabular}

* Change rate $=(2005,2006,2007$ prevalence-2004 prevalence $) / 2004$ prevalence $\mathrm{PMV}=$ prolonged mechanical ventilation

Hospital treatment cost was adjusted for specific hospital levels according to their differences in BNHI reimbursements. Cost-to-charge ratios were used to derive costs from hospital charges, and costs were inflation adjusted to 2007 dollars. Furthermore, to reflect changes in real dollar value, cost data were adjusted by the consumer price index for each year of 2004-2007 (95.45, 97.65, 98.23, and 100.00, respectively). Additionally, hospital treatment costs were then converted from Taiwan dollars to United States dollars, at an exchange rate of 31.0:1, which was the average exchange rate during 2004-2007. Statistical analyses were performed using statistics software (SPSS 15.0, SPSS, Chicago, Illinois). All tests were 2 -sided, and $P$ values $<.05$ were considered statistically significant.

\section{Results}

The prevalence rate of PMV patients was 94.3 per 100,000 persons in 2004, and gradually declined to 89.38 per 100,000 persons by 2007 . Thus, the rate of decline from 2004 to 2007 was $5.22 \%$, which was statistically significant $(P<.001)$ (Table 1$)$.

Regarding patient attributes, the estimated mean \pm SD age was $77.99 \pm 13.87$ years, and $37.37 \%$ of the PMV patients were female (Table 2). Furthermore, $0.56 \%$ of the PMV patients had a Charlson comorbidity index of 0 ; $1.49 \%$ had a Charlson comorbidity index of $1 ; 3.96 \%$ had a Charlson comorbidity index of $2 ; 9.41 \%$ had a Charlson comorbidity index of 3 ; and $84.59 \%$ had a Charlson comorbidity index $\geq 4$. In terms of patient referral source, the referring department was general medicine in $36.94 \%$ of patients, general surgery in $4.86 \%$, cardiology in $4.78 \%$, pulmonary in $44.94 \%$, neurology/neurosurgery in $5.89 \%$, and other in $2.59 \%$. Regarding hospital attributes, $34.65 \%$ of the PMV patients were treated in medical centers, $41.72 \%$ were treated in regional hospitals, and $25.11 \%$ were treated in district hospitals. In terms of volume, $50.71 \%$ of the PMV patients were treated in lowvolume centers, $24.18 \%$ were treated in medium-volume centers, and $36.94 \%$ were treated in high-volume centers.
Table 2. Characteristics of the Study Population $(n=65,181)$

\begin{tabular}{lc}
\hline \hline Age, median (IQR) y & $80(18-91)$ \\
Age Group, no. (\%) & \\
$<65$ y & $9,635(14.78)$ \\
$65-74$ y & $9,236(14.17)$ \\
$\geq 75$ y & $46,310(71.05)$ \\
Male, no. (\%) & $40,823(62.63)$ \\
Female, no. (\%) & $24,358(37.37)$ \\
Charlson Comorbidity Index score, & $4(0-6)$ \\
$\quad$ median (IQR) & \\
Charlson Comorbidity Index score, & \\
$\quad$ no. (\%) & \\
0 & \\
1 & $364(0.56)$ \\
2 & $969(1.49)$ \\
3 & $2,581(3.96)$ \\
$\geq 4$ & $6,133(9.41)$ \\
Patient referral source, no. (\%) & $55,134(84.59)$ \\
General medicine & \\
General surgery & $24,078(36.94)$ \\
Cardiology & $3,169(4.86)$ \\
Pulmonary & $3,114(4.78)$ \\
Neurology/neurosurgery & $29,294(44.94)$ \\
Other & $3,836(5.89)$ \\
Hospital level, no. (\%) & $1,690(2.59)$ \\
Medical center & \\
Regional hospital & \\
District hospital & $15,400(23.63)$ \\
Hospital volume, median (IQR) cases/y & $22,588(34.65)$ \\
Hospital volume, no. $\%)$ & $27,193(41.72)$ \\
$<300$ (low) & $418(24-1,853)$ \\
$300-999$ (medium) & \\
$\geq 1,000$ (high) & $16,368(25.11)$ \\
Age, mean \pm SD y & \\
Stay, mean \pm SD d & $33,052(50.71)$ \\
Hospital treatment costs, mean \pm SD & $15,761(24.18)$ \\
$\quad$ United States dollars & \\
\hline
\end{tabular}

Mean \pm SD stay was $33.26 \pm 22.52 \mathrm{~d}$, and mean $\pm \mathrm{SD}$ hospital treatment cost was $\$ 8,017.10 \pm \$ 5,877.53$.

Table 3 shows the results of trend analysis of patient and hospital attributes for each year from 2004 to 2007. The percentage of PMV patients $<65$ years old and 65 74 years old significantly increased, from $12.73 \%$ and $13.20 \%$ in 2004 to $16.50 \%$ and $15.22 \%$ by 2007 , respectively, but that of patients $\geq 75$ years old significantly decreased, from $74.06 \%$ to $68.27 \%$, during the same period $(P<.001)$. The percentage of female patients treated significantly decreased, from $37.73 \%$ in 2004 to $36.68 \%$ in $2007(P=.03)$. During the same period the percentage of patients with Charlson comorbidity index $\leq 2$ and 3 significantly decreased, from $6.86 \%$ and $10.20 \%$ to $4.54 \%$ and $8.01 \%$, respectively, but the percentage of patients with Charlson comorbidity index of 4 and $\geq 5$ significantly increased, from $58.22 \%$ and $24.72 \%$ to $61.67 \%$ and 
Table 3. Temporal Trends in Study Population Demographics and Hospital Data

\begin{tabular}{|c|c|c|c|c|c|}
\hline & $\begin{array}{c}2004 \\
(n=16,297)\end{array}$ & $\begin{array}{c}2005 \\
(n=16,278)\end{array}$ & $\begin{array}{c}2006 \\
(n=16,310)\end{array}$ & $\begin{array}{c}2007 \\
(n=16,296)\end{array}$ & $P$ \\
\hline \multicolumn{6}{|l|}{ Age Group, \% } \\
\hline$<65 \mathrm{y}$ & 12.73 & 14.76 & 15.13 & 16.50 & $<.001$ \\
\hline $65-74$ y & 13.20 & 13.85 & 14.40 & 15.22 & \\
\hline$\geq 75 \mathrm{y}$ & 74.06 & 71.38 & 70.47 & 68.27 & \\
\hline Male, $\%$ & 62.27 & 61.93 & 63.00 & 63.32 & .03 \\
\hline Female, $\%$ & 37.73 & 38.07 & 37.00 & 36.68 & \\
\hline \multicolumn{6}{|l|}{ Charlson Comorbidity Index, \% } \\
\hline$\leq 2$ & 6.86 & 6.77 & 5.85 & 4.54 & $<.001$ \\
\hline 3 & 10.20 & 10.52 & 8.90 & 8.01 & \\
\hline 4 & 58.22 & 59.74 & 61.18 & 61.67 & \\
\hline$\geq 5$ & 24.72 & 22.97 & 24.07 & 25.78 & \\
\hline \multicolumn{6}{|l|}{ Patient referral source, $\%$} \\
\hline General medicine & 48.41 & 37.30 & 30.94 & 31.11 & $<.001$ \\
\hline General surgery & 5.17 & 4.95 & 4.54 & 4.79 & \\
\hline Cardiology & 4.54 & 4.95 & 4.91 & 4.71 & \\
\hline Pulmonary & 33.65 & 44.48 & 51.69 & 49.95 & \\
\hline Neurology & 5.60 & 5.98 & 5.58 & 6.38 & \\
\hline Other & 2.62 & 2.33 & 2.34 & 3.07 & \\
\hline \multicolumn{6}{|l|}{ Hospital level, \% } \\
\hline Medical center & 20.08 & 23.20 & 23.05 & 28.17 & $<.001$ \\
\hline Regional hospital & 44.56 & 42.92 & 29.55 & 21.59 & \\
\hline District hospital & 35.35 & 33.88 & 47.39 & 50.23 & \\
\hline \multicolumn{6}{|l|}{ Hospital volume, \% } \\
\hline$\leq 25$ (low) & 27.99 & 21.46 & 28.37 & 22.62 & $<.001$ \\
\hline 26-74 (medium) & 57.69 & 60.95 & 41.85 & 42.36 & \\
\hline$\geq 75$ (high) & 14.32 & 17.59 & 29.78 & 35.02 & \\
\hline Stay, mean \pm SD d & $35.12 \pm 24.24$ & $33.82 \pm 23.26$ & $32.47 \pm 21.59$ & $31.61 \pm 20.64$ & $<.001$ \\
\hline Hospital treatment cost, mean \pm SD dollars & $7,933.17 \pm 5,798.83$ & $7,995.41 \pm 6,208.93$ & $7,882.41 \pm 5,543.85$ & $8,257.52 \pm 5,933.02$ & $<.001$ \\
\hline
\end{tabular}

$25.78 \%$, respectively $(P<.001)$. The percentages of patients referred from general medicine and general surgery departments decreased, from $48.41 \%$ and $5.17 \%$ to $31.11 \%$ and $4.79 \%$, respectively, but the percentages of patients referred from cardiology, pulmonary, neurology/neurosurgery, and other departments significantly increased $(4.54 \%$, $33.65 \%, 5.60 \%$, and $2.62 \%$ to $4.71 \%, 49.95 \%, 6.38 \%$, and $3.07 \%$, respectively, $P<.001$ ). In terms of hospital attributes, the percentage of patients treated in medical centers and district hospitals significantly increased, from $20.08 \%$ and $35.35 \%$ to $28.17 \%$ and $50.23 \%$, respectively, but the percentage of patients treated in regional hospitals significantly decreased from $44.56 \%$ to $21.59 \%(P<.001)$. Additionally, the percentage of patients treated in lowvolume and medium-volume centers significantly decreased, from $27.99 \%$ and $57.69 \%$ to $22.62 \%$ and $42.36 \%$, respectively, but the percentage of patients treated in high-volume centers significantly increased, from $14.32 \%$ to $35.02 \%(P<.001)$. Stay significantly decreased, from 35.12 days to 31.61 days, but hospital treatment cost significantly increased, from $\$ 7,933.17$ to $\$ 8,257.52$ $(P<.001)$.
Table 4 shows the data obtained by multiple regression models used to evaluate the predictors of stay. These data indicated that high Charlson comorbidity index $(P<.001)$, patient referral from general medicine department $(P<.001)$, treatment at a regional or district hospital $(P<.001)$, and treatment in a medium- or high-volume hospital $(P<.001)$ were significantly associated with short stay. Compared to patient attributes and hospital attributes, stay had a significantly larger coefficient with high-volume centers compared to low- or medium-volume centers (regression coefficient $-20.14 \mathrm{~d}$, $P<.001)$.

Table 5 shows how data obtained by multiple regression models were used to evaluate the predictors of hospital treatment costs. These data indicated that advanced age $(P<.001)$, low Charlson comorbidity index $(P<.001)$, patient referral from pulmonary department $(P<.001)$, treatment at a regional or district hospital $(P<.001)$, treatment at a high-volume hospital $(P<.001)$, and short stay $(P<.001)$ were associated with decreased hospital treatment costs. Compared to patient attributes and hospital attributes, hospital treatment costs had a significantly larger 
Trend Analysis of Hospital Resource Utilization for Prolonged Mechanical Ventilation

Table 4. Multiple Regression Model of the Relationship Between Effective Predictors and Stay for Patients on Prolonged Mechanical Ventilation

\begin{tabular}{|c|c|c|c|}
\hline & Coefficient & $\begin{array}{l}\text { Standard } \\
\text { Error }\end{array}$ & $P$ \\
\hline Constant & 63.18 & & $<.001$ \\
\hline \multicolumn{4}{|l|}{ Year } \\
\hline 2004 & Reference & & \\
\hline 2005 & -4.24 & -0.08 & $<.001$ \\
\hline 2006 & -8.67 & -0.15 & $<.001$ \\
\hline 2007 & -5.18 & -0.09 & $<.001$ \\
\hline Age & 0.03 & 0.02 & .24 \\
\hline \multicolumn{4}{|l|}{ Sex } \\
\hline Male & Reference & & \\
\hline Female & 0.29 & 0.02 & .62 \\
\hline \multicolumn{4}{|c|}{ Charlson Comorbidity Index } \\
\hline$\leq 2$ & Reference & & \\
\hline 3 & -14.11 & -0.25 & $<.001$ \\
\hline 4 & -13.57 & -0.24 & $<.001$ \\
\hline$\geq 5$ & -14.34 & -0.26 & $<.001$ \\
\hline \multicolumn{4}{|l|}{ Patient referral source } \\
\hline General medicine & Reference & & \\
\hline General surgery & 2.79 & 0.03 & .14 \\
\hline Cardiology & 2.34 & 0.02 & .34 \\
\hline Pulmonary & 8.89 & 0.18 & $<.001$ \\
\hline Neurology & 0.38 & 0.02 & .57 \\
\hline Other & 4.23 & 0.03 & .10 \\
\hline \multicolumn{4}{|l|}{ Hospital level } \\
\hline Medical center & Reference & & \\
\hline Regional hospital & -7.47 & -0.15 & $<.001$ \\
\hline District hospital & -6.69 & -0.14 & $<.001$ \\
\hline \multicolumn{4}{|l|}{ Hospital volume } \\
\hline$\leq 25$ (low) & Reference & & \\
\hline 26-74 (medium) & -13.54 & -0.31 & $<.001$ \\
\hline$\geq 75$ (high) & -20.14 & -0.34 & $<.001$ \\
\hline
\end{tabular}

coefficient with stay (regression coefficient $\$ 124.54$, $P<.001)$.

\section{Discussion}

This population-based study is the first to examine how patient attributes and hospital attributes reflect changing trends in the prevalence of PMV patients, and the first to identify factors that predict stay and hospital treatment costs. The present study confirmed the findings of previous studies that age $<74$ years, male sex, Charlson comorbidity index of 4 and higher, patient referral from cardiology, pulmonary, or neurology/neurosurgery department, treatment in medical center or district hospital, and treatment in high-volume hospital were associated with increased likelihood of PMV. Older patients who are predominantly male and with greater Charlson comorbidity index are the most likely to have longer stay and hos-
Table 5. Multiple Regression Model of the Relationship Between Effective Predictors and Hospital Treatment Costs for Prolonged Mechanical Ventilation Patients

\begin{tabular}{|c|c|c|c|}
\hline Variables & Coefficient & $\begin{array}{l}\text { Standard } \\
\text { Error }\end{array}$ & $P$ \\
\hline Constant & $6,558.17$ & & $<.001$ \\
\hline \multicolumn{4}{|l|}{ Year } \\
\hline 2004 & Reference & & \\
\hline 2005 & $1,148.18$ & 0.08 & $<.001$ \\
\hline 2006 & $1,023.57$ & 0.07 & $<.001$ \\
\hline 2007 & $1,463.24$ & 0.11 & $<.001$ \\
\hline Age & -13.22 & -0.04 & $<.001$ \\
\hline \multicolumn{4}{|l|}{ Sex } \\
\hline Male & Reference & & \\
\hline Female & -270.43 & -0.02 & 0.06 \\
\hline \multicolumn{4}{|c|}{ Charlson Comorbidity Index } \\
\hline$\leq 2$ & Reference & & \\
\hline 3 & 998.27 & 0.07 & $<.001$ \\
\hline 4 & $1,063.61$ & 0.08 & $<.001$ \\
\hline$\geq 5$ & $1,057.31$ & 0.08 & $<.001$ \\
\hline \multicolumn{4}{|l|}{ Patient referral source } \\
\hline General medicine & Reference & & \\
\hline General surgery & $5,018.09$ & 0.19 & $<.001$ \\
\hline Cardiology & $1,422.51$ & 0.04 & .002 \\
\hline Pulmonary & $-1,329.47$ & -0.12 & $<.001$ \\
\hline Neurology & $1,125.83$ & 0.06 & $<.001$ \\
\hline Other & $1,644.30$ & 0.04 & $<.001$ \\
\hline \multicolumn{4}{|l|}{ Hospital level } \\
\hline Medical center & Reference & & \\
\hline Regional hospital & $-5,100.18$ & -0.39 & $<.001$ \\
\hline District hospital & $-6,049.78$ & -0.42 & $<.001$ \\
\hline \multicolumn{4}{|l|}{ Hospital volume } \\
\hline$\leq 25$ (Low) & Reference & & \\
\hline 26-74 (Medium) & -778.57 & 0.09 & $<.001$ \\
\hline$\geq 75$ (High) & $-1,430.32$ & -0.11 & $<.001$ \\
\hline Stay & 124.54 & 0.53 & $<.001$ \\
\hline
\end{tabular}

pital treatment costs, probably because these characteristics represent the most frail and sick patients.

National registry studies such as those performed by the BNHI in Taiwan are an excellent source of populationbased data for evaluating the current practice of PMV. ${ }^{13-15}$ Unlike single-center series studies, data from registry studies provide an overview of practices in large populations while avoiding referral bias or bias reflecting the practices of individual physicians or institutions. ${ }^{13-15}$

There are few females in the population of patients receiving PMV. In 2010 the average life expectancy of the Taiwanese population for males and females was 78 years and 83 years, respectively. We hypothesize that the higher rate in males results from lower Charlson comorbidity index and the tendency of diseases to be more severe in males than in females at the time of medical intervention, because males tend to delay medical treatment. ${ }^{16}$ 
The incidence and prevalence of PMV depends on the specific setting and on how PMV is defined. No multicenter studies have applied a strict definition of PMV such as 21 days or longer. However, single-site studies indicate that $3-7 \%$ of patients receiving mechanical ventilation meet such criteria. ${ }^{11}$ Population-based studies of PMV often use the former Diagnosis-Related Group 483 to identify patients in large administrative databases. ${ }^{17}$ An earlier analysis of a statewide North Carolina database between 1993 and 2002 revealed a $78 \%$ increase in the number of patients discharged with Diagnosis-Related Group 483 after receiving mechanical ventilation (ie, from 43.2/1,000 such patients in 1993 to $77.1 / 1,000$ patients in 2002). ${ }^{3}$ In our study the prevalence of PMV patients per 100,000 patients declined gradually, from 94.30 in 2004 to 89.38 in 2007. Based on the BNHI policy requiring quality of care for PMV patients, the total numbers of PMV patients has not increased, but the total population of Taiwan increased. ${ }^{18}$ Our study also showed a significant decrease in the prevalence of PMV in older patients (ie, PMV patients age 75 years or older decreased from $74 \%$ in 2004 to $68 \%$ in 2007 , but the number of comorbidities also increased from 2004 to 2007 during the same period). Duration of ventilation is reportedly associated with cause of ICU admission, age, and treatment location. ${ }^{19}$ Most PMV patients in the current study had been referred from pulmonary departments, and the percentage of patients, from 33.65\% in 2004 to $49.95 \%$ in 2007 , is consistent with previous studies. ${ }^{4,20}$

The temporal trend showed that a higher Charlson comorbidity index was observed from 2004 to 2007, but the stay trend was reduced during the same time period. This counterintuitive association also explains that patients with greater comorbidity are dying in hospitals. This deserves a further study in the near future.

Stay also decreased, from 35.12 days in 2004 to 31.61 days in 2007. The 2000 BNHI policy for PMV patients apparently decreased the stay of PMV patients. ${ }^{18}$ The decrease in stay was larger in regional hospitals and district hospitals, as compared to medical centers, and most patients had initiated mechanical ventilation in the ICUs of short-term acute care medical centers. Patients with underlying chronic cardiorespiratory illnesses tended to remain ventilator-dependent and tended to transition from acute illness status to chronic or critical illness status. During this transition, alternatives to ICU care in medical centers should be considered. The literature shows that patients treated in high-volume medical centers have significantly shorter stay, compared to those treated in lowvolume centers. ${ }^{21}$ The time required before resuming work or other normal activity is also reportedly longer in patients treated in low-volume centers, compared to those treated in high-volume centers, which is consistent with the observation in this study that patients treated in high- volume centers had shorter hospital stays than those treated in low-volume centers did.

An analysis by the Medicare Payment Advisory Commission indicated that the treatment costs for PMV patients can be reduced by early transfer to regional or district hospitals. ${ }^{22}$ The data revealed that hospital treatment costs for patients receiving PMV increased from $\$ 7,933.17$ in 2004 to $\$ 8,257.52$ in 2007 . The data also reflect changing trends of PMV. A higher Charlson comorbidity index and treatment at a medical center were positively associated with hospital treatment costs. This perhaps explains why the hospital treatment costs were going up during the study period. ${ }^{5,22,23}$

In the study, the rising costs, shorter stay, and a decreased temporal trend of prevalence of PMV patients, especially for an aging population with fewer older age individuals, were simultaneously observed over time. These results are the opposite of those in the United States, which show that the incidence of PMV patients being discharged to long-term acute care hospitals is increasing. ${ }^{24}$ In Taiwan, there was an increased percentage of patients coming from high-volume medical centers during the same time period.

Efforts to slow the growth in hospital treatment costs have increased scrutiny of various patient subgroups in order to minimize unnecessary treatments and to limit lifesustaining therapies that do not benefit the patient, such as in cases of brain death. Our analyses may help to develop guidelines for timing the changes in care for PMV patients. Increasing nurse-to-patient ratios in respiratory care centers and respiratory care wards to ratios exceeding those in ICUs may also decrease hospital treatment costs.

\section{Limitations}

This study has several limitations that are inherent in any large database analysis. First, the clinical picture obtained by analyzing claims data are not as precise as that obtained by analyzing prospective clinical trial data, due to possible errors in the coding of primary diagnoses and treatment modalities. Additionally, the relationship between these factors and hospital resource utilization could not be evaluated. Second, complications associated with hospital resource utilization were not assessed, which limits the validity of the predictions. Finally, the analysis did not examine outcome data such as patient-reported quality of life and indirect costs incurred after discharge.

\section{Conclusions}

In conclusion, these population-based data demonstrated a decrease in the prevalence of PMV, especially for older patients, and it also showed that stay decreased; however, hospital treatment costs increased. Healthcare providers 
and patients should understand that stay and hospital treatment costs depend not only on patient attributes but also on hospital attributes.

\section{ACKNOWLEDGMENTS}

For assistance with statistical analyses, the authors thank Tsu-Nai Wang, Statistical Analysis Laboratory, Department of Medical Research, Kaohsiung Medical University Hospital, Kaohsiung Medical University.

\section{REFERENCES}

1. Bennett S, Hurford WE. When should sedation or neuromuscular blockade be used during mechanical ventilation? Respir Care 2011; 56(2):168-176.

2. Williams K, Hinojosa-Kurtzberg M, Parthasarathy S. Control of breathing during mechanical ventilation: who is the boss? Respir Care 2011;56(2):127-136.

3. Estenssoro E, González F, Laffaire E, Canales H, Sáenz G, Reina R, Dubin A. Shock on admission day is the best predictor of prolonged mechanical ventilation in the ICU. Chest 2005;127(2):598-603.

4. Brunello AG, Haenggi M, Wigger O, Porta F, Takala J, Jakob SM. Usefulness of a clinical diagnosis of ICU-acquired paresis to predict outcome in patients with SIRS and acute respiratory failure. Intensive Care Med 2010;36(1):66-74.

5. Unroe M, Kahn JM, Carson SS, Govert JA, Martinu T, Sathy SJ, et al. One-year trajectories of care and resource utilization for recipients of prolonged mechanical ventilation: a cohort study. Ann Intern Med 2010;153(3):167-175.

6. White AC, Joseph B, Gireesh A, Shantilal P, Garpestad E, Hill NS, O'Connor HH. Terminal withdrawal of mechanical ventilation at a long-term acute care hospital: comparison with a medical ICU. Chest 2009;136(2):465-470.

7. Su J, Lin CY, Chen PJ, Lin FJ, Chen SK, Kuo HT. Experience with a step-down respiratory care center at a tertiary referral medical center in Taiwan. J Crit Care 2006;21(2):156-161.

8. Divo MJ, Murray S, Cortopassi F, Celli BR. Prolonged mechanical ventilation in Massachusetts: the 2006 prevalence survey. Respir Care 2010;55(12):1693-1698.

9. Deyo RA, Cherkin DC, Ciol MA. Adapting a clinical comorbidity index for use with ICD-9-CM administrative databases. Clin Epidemiol 1992;45(6):613-619.

10. Mamidanna R, Bottle A, Aylin P, Faiz O, Hanna GB. Short-term outcomes following open versus minimally invasive esophagectomy for cancer in England: a population-based national study. Ann Surg 2012;255(2):197-203.

11. Mnatzaganian G, Ryan P, Norman PE, Hiller JE. Accuracy of hospital morbidity data and the performance of comorbidity scores as predictors of mortality. J Clin Epidemiol 2012;65(1):107-115.
12. Devon KM, Urbach DR, McLeod RS. Postoperative disposition and health services use in elderly patients undergoing colorectal cancer surgery: a population-based study. Surgery 2011;149(5):705-712.

13. Cox CE, Carson SS, Lindquist JH, Olsen MK, Govert JA, Chelluri L; Quality of Life After Mechanical Ventilation in the Aged (QOL-MV) investigators. Differences in one-year health outcomes and resource utilization by definition of prolonged mechanical ventilation: a prospective cohort study. Crit Care 2007;11(1):R9.

14. Kojicic M, Li G, Ahmed A, Thakur L, Trillo-Alvarez C, Cartin-Ceba $\mathrm{R}$, et al. Long-term survival in patients with tracheostomy and prolonged mechanical ventilation in Olmsted County, Minnesota. Respir Care 2011;56(11):1765-1770.

15. Scala R, Corrado A, Confalonieri M, Marchese S, Ambrosino N; Scientific Group on Respiratory Intensive Care of the Italian Association of Hospital Pneumologists. Increased number and expertise of Italian respiratory high-dependency care units: the second national survey. Respir Care 2011;56(8):1100-1107.

16. Nieuwenhuis MM, Jaarsma T, van Veldhuisen DJ, van der Wal MH. Factors associated with patient delay in seeking care after worsening symptoms in heart failure patients. J Card Fail 2011;17(8):657-663.

17. Dewar DM, Kurek CJ, Lambrinos J, Cohen IL, Zhong Y. Patterns in costs and outcomes for patients with prolonged mechanical ventilation undergoing tracheostomy: an analysis of discharges under diagnosis-related group 483 in New York State from 1992 to 1996. Crit Care Med 1999;27(12):2640-2647.

18. Wang YH, Lin AS, Chao TY, Lu SN, Liu JW, Chen SS, Lin MC. A cluster of patients with severe acute respiratory syndrome in a chest ward in southern Taiwan. Intensive Care Med 2004;30(6):1228-1231.

19. Epstein SK, Vuong V. Lack of influence of gender on outcomes of mechanically ventilated medical ICU patients. Chest 1999;116(3): 732-739.

20. Jubran A, Lawm G, Duffner LA, Collins EG, Lanuza DM, Hoffman LA, Tobin MJ. Post-traumatic stress disorder after weaning from prolonged mechanical ventilation. Intensive Care Med 2010;36(12): 2030-2037.

21. Urbach DR, Baxter NN. Does it matter what a hospital is "high volume" for? Specificity of hospital volume-outcome associations for surgical procedures: analysis of administrative data. BMJ 2004; 328(7442):737-740.

22. Wunsch H, Linde-Zwirble WT, Angus DC, Hartman ME, Milbrandt EB, Kahn JM. The epidemiology of mechanical ventilation use in the United States. Crit Care Med 2010;38(10):1947-1953.

23. Carson SS, Cox CE, Holmes GM, Howard A, Carey TS. The changing epidemiology of mechanical ventilation: a population-based study. J Intensive Care Med 2006;21(3):173-182.

24. Kahn JM, Benson NM, Appleby D, Carson SS, Iwashyna TJ. Longterm acute care hospital utilization after critical illness. JAMA 2010; 303(22):2253-2259. 